
\title{
28 Research Suare \\ Effects of Resuming Training Using Yoga on Physical Functions and Fitness of Taekwondo Athletes After High-intensity Training
}

Feng Liu ( $\sim$ liufeng_1981@126.com )

Henan University

Gaixia Hou

Henan University

Cheng Liang

Anyang Institute of Technology

\section{Research}

Keywords: High-intensity, Yoga, Resuming Training, Taekwondo Athletes, Physical Function, Physical Fitness

Posted Date: August 3rd, 2020

DOI: https://doi.org/10.21203/rs.3.rs-42532/v1

License: (c) (i) This work is licensed under a Creative Commons Attribution 4.0 International License. Read Full License 


\section{Abstract}

Objective: This study aimed to analyze the effects of resuming training using yoga on the physical functions and fitness of Taekwondo athletes after high-intensity training.

Method: A total of 50 Taekwondo athletes from this college were randomly divided into 2 groups. 25 athletes are in the control group, and they massage their muscles after high-intensity training to help recovery. Meanwhile, 25 athletes are in observation groups, and they practice yoga after high-intensity training for resuming training. After 8 weeks, the physical functions and fitness of the two groups of athletes were compared.

Result: There was no statistically significant difference between the observation group and the control group before starting high-intensity training from 1 st week to 8 th week $(P<0.05)$. From the 4 th week to the 8th week after high-intensity training, the observation group's heart rate, heart rate after resuming training and subjective physical sensation degree scores were lower than the control group $(P<0.05)$. After 8 weeks of resuming training in the observation group, hemoglobin was higher than the control group, blood urea nitrogen and creatine kinase was lower than the control group $(P<0.05)$. The observation group's flexion test results of the sitting position after 4th week and 8th week of resuming training were higher than those of the control group $(P<0.05)$.

Conclusion: The use of yoga for resuming training after high-intensity training can improve the physical function and fitness of Taekwondo athletes, improve the flexibility of athletes and promote their professional skills.

\section{Background}

Professional Taekwondo athletes have a high level of competition, and contests between athletes must ensure good physical reserve, so as to ensure that their skills are fully displayed on the competition field. Athletes' physical reserve must go through the process of exercise stimulation, fatigue, recovery, and adaptation, and the athlete's physical fitness can be recovered excessively after the process is completed. Athletes generate fatigue after exercise stimulation. In this case, if the fatigue is not eliminated in time, athletes will not be able to adapt to further training load, which will eventually have an adverse effect on the training results [1-2].

Taekwondo training has a large amount of exercise, high intensity, and needs to be repeated continuously. It belongs to a type of periodic exercise. If the resuming training is not done after each training, fatigue is easy to accumulate, and the body will not adapt to the training for a long time. Athletes will not be able to complete the prescribed training content, which will affect the athlete's athletic ability [3]. For the recovery of high-intensity training, athletes generally choose some moderate and light-load training to achieve the relief of exercise fatigue. Alternating the large load of training with the moderate and low load of recovery training can make different metabolites interact with each other, thereby promoting a better metabolism of a party [4]. In the past, the commonly used restorative methods mostly 
used jogging, stretching, and ice compress. However, the application effect of a single method is not outstanding, and it usually takes a long time to harvest the effect, and it is necessary to alternately perform different methods to recover [5].

Recently, Henan Taekwondo team has gradually tried yoga training after high-intensity training of Taekwondo to help recovery. Yoga, as a movement to regulate the state of mind and body, is mainly carried out by adjusting breathing and performing posture exercises. Many studies on the physiological and psychological effects of yoga on the body suggest positive conclusions [6]. There is not much research on whether yoga is effective as a small-intensity recovery training method after high-intensity training. This study specifically analyzed 50 Taekwondo athletes of Henan Taekwondo team in training, and provided new directions for the selection of Taekwondo athletes' recovery training methods after training.

\section{Material And Methods}

\section{Materials}

In 2019, the Henan Taekwondo team selected 50 male athletes in training as the subjects of this study. They were divided into 2 groups by random drawing method, and there are 25 athletes in control group and observation group, separately. Before participating in this study, all athletes participated in the training normally, and their activities, life patterns, and emotional states were not abnormal. And all the athletes have received professional taekwondo training for more than 2 years, and have no health problems before participating in the study.

\section{Methods}

All athletes in the two groups receive training with the same exercise intensity and the same amount of exercise every day according to the training plan. The control group massaged their muscles after each training session to help relax for 20 minutes. The observation group received yoga rehabilitation training after each training session for 20 minutes.

Method of control group. The players are divided into groups of two. One of them is lying on a yoga mat, the other one is stepping on the muscles of the whole body including the legs, waist, back, and hands with his feet, and the two players exchange after a sense of relaxation.

Method of observation group. Yoga preparation: use 5 minutes to adjust your breathing yourself. Yoga practice: Choose forward bending sitting, one leg side stretching, swinging, big worship, supine twisting, crescent and horseback twisting, pulling feet, leg extension, pigeon style and neck extension for practice[7] for ten minutes. End of Yoga: Keep lying on your back for 5 minutes. You should pay attention to the following two points during yoga training. The first is that the selected yoga action must be combined with the characteristics of Taekwondo. And it is necessary to focus on the stretching of the 
ankle, hip, shoulder, and waist for the specific fatigued parts of the athlete after the training. The second is that during yoga training, all postures must not cause discomfort to the athletes. During the training, guide the athletes to breathe steadily and experience the feeling of body feedback.

\section{Observation Index}

Physical function: Determine the heart rate, subjective physical sensation, hemoglobin, blood urea nitrogen and creatine kinase levels of the two groups of athletes. The heart rate needs to be measured before training, immediately after high-intensity training, and after recovery. The degree of subjective physical sensation is completed using the simplified version of the subjective physical sensation table [8], which is divided into 10 levels. Level 0 means no feeling, level 1 and level 2 means weak, level 3 means mild, level 4 means slightly stronger, level 5 means strong, level 6 means medium, and level 7 means very strong, level 8, level 9 and level 10 means very strong. The scores are $0-10$, respectively. The higher the score, the worse the physical strength. The levels of hemoglobin, blood urea nitrogen, and creatine kinase was measured after the test of $2 \mathrm{ml}$ of elbow vein blood collected by the team doctor under sterile conditions.

Physical fitness: Considering that the flexibility of Taekwondo athletes is very important, this study adopts flexibility as an evaluation index of athletes' physical fitness. Specifically, measure the flexion distance of the sitting position. Each athlete performs two sitting flexion tests to record the final result with better results.

\section{Statistical Method}

Statistical analysis was performed with SPSS23.0, and measurement data was expressed as ( \pm s), and results were compared using independent sample t test. Counting data is represented by [n(\%)], X2 test is used for comparison of results, ANVOA is used for multi-point comparison within the group, $\mathrm{F}$ test is used, graphs are made by Graphpad Prism 8. If $\mathrm{P}<0.05$, the difference is considered statistically significant.

\section{Results}

\section{Physical Data}

The average age, height, body weight, body mass index (BMI) and training time of the 25 athletes in the observation group and the 25 athletes in the control group were not significantly different, and there was no statistical significance $(P>0.05)$ (Table 1$)$.

Table 1 Comparison of 2 groups of physical data $\square \pm \mathbf{s} \square$ 


\begin{tabular}{|c|c|c|c|c|}
\hline Data & Observation group $(\mathrm{n}=25 \rrbracket$ & Control group $(n=25)$ & $t$ & $P$ \\
\hline Average age $\$ year $\rrbracket$ & $20.46 \pm 1.13$ & $20.51 \pm 1.18$ & 0.153 & 0.879 \\
\hline Average height $\ \mathrm{~cm} \rrbracket$ & $185.43 \pm 2.61$ & $186.49 \pm 2.37$ & 1.503 & 0.139 \\
\hline Average body weight $₫ \mathrm{~kg} \rrbracket$ & $70.52 \pm 3.68$ & $70.48 \pm 3.71$ & 0.038 & 0.970 \\
\hline $\mathrm{BMI}\left(\mathrm{kg} / \mathrm{m}^{2}\right)$ & $20.60 \pm 1.39$ & $20.43 \pm 1.45$ & 0.423 & 0.674 \\
\hline Average training time $₫ y e a r \rrbracket$ & $2.83 \pm 0.56$ & $2.76 \pm 0.53$ & 0.464 & 0.652 \\
\hline
\end{tabular}

\section{Heart Rate Before Training}

The heart rate level of the observation group before starting high-intensity training from 1 st week to 8th week only fluctuated about $1 \mathrm{time} / \mathrm{min}$, and the heart rate comparison between different times showed no statistical difference $(P>0.05)$. The heart rate of the control group fluctuated only about 2 times $/ \mathrm{min}$ before the beginning of high-intensity training from 1 st week to 8 th week. The comparison of heart rate between different times did not show statistical difference $(P>0.05)$. There was no statistically significant difference between the observation group and the control group before starting high-intensity training from 1 st week to 8 th week $(P<0.05)($ Table 2).

Table 2 Heart rate analysis before training for two groups in 8 weeks $\square \pm \mathbf{s} \square$

\begin{tabular}{|lllll|}
\hline Time & Observation group $(\mathrm{n}=25 \rrbracket$ & Control group $(\mathrm{n}=25)$ & $t$ & $P$ \\
\hline The first week & $73.52 \pm 4.16$ & $73.95 \pm 5.04$ & 0.329 & 0.744 \\
\hline The second week & $73.59 \pm 4.58$ & $73.19 \pm 5.27$ & 0.286 & 0.776 \\
\hline The third week & $73.29 \pm 4.62$ & $74.18 \pm 4.52$ & 0.688 & 0.495 \\
\hline The fourth week & $74.19 \pm 5.31$ & $74.18 \pm 3.55$ & 0.008 & 0.994 \\
\hline The fifth week & $74.19 \pm 5.34$ & $75.26 \pm 6.39$ & 0.642 & 0.524 \\
\hline The sixth week & $73.26 \pm 3.62$ & $74.18 \pm 3.83$ & 0.873 & 0.387 \\
\hline The seventh week & $73.96 \pm 4.26$ & $74.18 \pm 5.02$ & 0.167 & 0.868 \\
\hline The eighth week & $73.61 \pm 5.19$ & $74.18 \pm 4.27$ & 0.424 & 0.673 \\
$t$ & 0.578 & 0.635 & & \\
\hline$P$ & 0.127 & 0.327 & & \\
\hline
\end{tabular}




\section{Instant Heart Rate After High-intensity Training}

There was no statistically significant difference in the instant heart rate between the observation group and the control group at $1 \mathrm{st}, 2 \mathrm{nd}$, and 3rd week after high-intensity training $(P>0.05)$. There was no statistically significant difference in the instant heart rate in the observation group from 1st week to 8th week after high-intensity training $(P>0.05)$. There was no statistically significant difference in the instant heart rate in the control group from 1 st week to 8 th week after high-intensity training $(P>0.05)$. The observation group's instant heart rate from 4th week to 8th week after high-intensity training was lower than that of the control group $(\mathrm{P}<0.05)$ (Figure 1).

Compared with the control group's instant heart rate after 1st week, 2nd week, and 3rd week after highintensity training, the observation group showed no significant difference $(P>0.05)$. Compared with the instant heart rate after high-intensity training at 4th, 5th, 6th, 7th, and 8th week in the control group, the observation group was lower $(\mathrm{P}<0.05)$. ${ }^{*}$ Indicates $\mathrm{P}<0.05$ between the two groups.

\section{Heart Rate After Resuming Training}

There was no statistically significant difference in heart rate between the observation group and the control group after the resuming training at 1 st, $2 n d$, and $3 r d$ week $(P>0.05)$. There was no statistically significant difference in the heart rate comparison of observation group from 1st week to 8th week after high-intensity training $(P>0.05)$. There was no statistically significant difference in the heart rate comparison of control group from 1 st week to 8th week after the recovery training $(P>0.05)$. The heart rate of the observation group was lower than that of the control group from the 4th week until the end of the 8th week of resuming training $(P<0.05)$ (Figure 2).

Compared with the heart rate of the control group after 1st week, 2nd week, and 3rd week of resuming training, the observation group had no significant difference $(P>0.05)$. Compared with the heart rate of the control group after 4th, 5th, 6th, 7th, and 8th week of resuming training, the observation group was lower $(\mathrm{P}<0.05)$. * Indicates $\mathrm{P}<0.05$ between the two groups.

\section{Subjective Physical Sensation}

There was no statistically significant difference between the observation group and the control group in the scores of subjective physical sensation degree at 1 st week, 2 nd week, and 3rd week $(P>0.05)$. There was a statistically significant difference in the subjective physical sensation degree scores in the observation group from 1 st week to 8 th week $(P<0.05)$. There was no statistically significant difference in the scores of subjective physical sensation between the control group for adjacent 2 weeks. From the 4 th 
week to the 8th week, the observation group's subjective physical sensation score was lower than that of the control group $(P<0.05)$ (Figure 3).

Compared with the scores of subjective physical sensation after 1 st week, 2nd week, and 3rd week of resuming training in the control group, the observation group showed no significant difference $(P>0.05)$. Compared with the scores of subjective physical sensation after 4th, 5tth, 6th, 7th, and 8th week of resuming training in the control group, the observation group was lower $(P<0.05) .{ }^{*}$ Indicates $P<0.05$ between the two groups.

\section{Biochemical Index}

There was no significant difference in the levels of biochemical indexes hemoglobin, blood urea nitrogen, and creatine kinase before the resuming training between the observation group and the control group $(P>0.05)$. After 8 weeks of resuming training, the observation group had a slight increase in hemoglobin, a slight decrease in blood urea nitrogen, and a slight decrease in creatine kinase. Meanwhile, in the control group, hemoglobin decreased slightly, blood urea nitrogen increased slightly, and creatine kinase increased slightly. However, there was no statistically significant difference between the two groups before and after resuming training $(P>0.05)$. After 8 weeks of resuming training, the observation group had higher hemoglobin than the control group, blood urea nitrogen and creatine kinase was lower than the control group $(P<0.05)$ (Figure 4).

Compared with the level of hemoglobin, blood urea nitrogen, and creatine kinase before the start of resuming training in the control group, there was no statistical difference in the observation group $(P>0.05)$. Compared with the control group after 8 weeks of resuming training, the observation group had higher level of hemoglobin and lower level of blood urea nitrogen and creatine kinase $(P<0.05)$. * Indicates $\mathrm{P}<0.05$ between the two groups.

\section{Physical Fitness}

Before the start of resuming training, the sitting body flexion test had no statistically significant difference between the observation group and the control group $(P>0.05)$. After 4 th week and 8th week of resuming training, the results of the sitting flexion test of the two groups were gradually increased, and the difference between the groups at different times was statistically significant $(P<0.05)$. After 4 th week and 8th week of resuming training, the observation group flexion test results were higher than the control group $(\mathrm{P}<0.05)($ Table 3$)$.

Table 3 Analysis of the results of the sitting body flexion test of the two groups before and after the resuming training $\square \pm \mathrm{s} \bigotimes \mathrm{cm} \rrbracket$ 


\begin{tabular}{|lllll|}
\hline Group & Number & Before resuming training & 4th week & 8th week \\
\hline observation group & 25 & $18.26 \pm 2.23$ & $21.13 \pm 1.97 *$ & $25.34 \pm 1.02 * \&$ \\
\hline control group & 25 & $18.31 \pm 2.29$ & $19.01 \pm 1.35$ & $22.16 \pm 1.24 * \&$ \\
\hline$t$ & & 0.078 & 4.439 & 9.903 \\
\hline$P$ & 0.938 & 0.000 & 0.000 \\
\hline
\end{tabular}

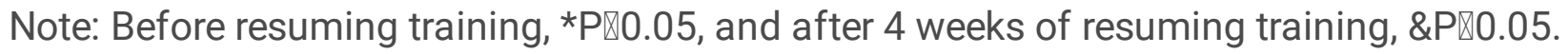

\section{Conclusion}

The heart rate before training measures the heart rate of the athlete in the resting state before starting high-intensity training. The normal level is between $45-80$ beats per minute. Generally, the lower the athlete's heart rate in the resting state, the stronger the heart function, the stronger the tolerance to training, and the better the body function. On the contrary, it reminds athletes that they lack the adaptability to the training load, which is easy to accumulate fatigue [9]. After high-intensity training, the instant heart rate is the exercise rate, and the highest heart rate of Taekwondo athletes under highintensity exercise is usually between $180-200$ beats/min [10]. The level of the athlete's heart rate during exercise is related to the intensity of training. Under the same exercise load, the slower the athlete's heart rate rises, the better his physical function. The lower the maximum heart rate of an athlete who receives the same intensity training, the better the athlete's physical function [11]. The heart rate measured after the athlete completing their respective resuming training is heart rate after resuming training. Under the same exercise intensity, the faster the heart rate level decreases after resuming training, the better the cardiovascular function, the faster and better the body recovery speed [12]. This study showed that the observation group and the control group had no significant difference in heart rate levels from the first week to the eighth week before starting high-intensity training. However, from the 4th week to the 8th week after high-intensity training, the instant heart rate and the heart rate after the resuming training in the observation group were both lower than the control group $(P<0.05)$. This indicated that the athletes in the observation group slowed down their maximum heart rate during high-intensity exercise after receiving yoga for resuming training. Compared with the control group, the observation group has better physical function, and the heart rate recovers more quickly after receiving yoga for resuming training. It shows that the athletes in the observation group have stronger heart reserve capacity. After the highintensity training, the athlete's body function can be recovered more quickly, and the athlete can better adapt to the training load. As can be seen from the above results, yoga can play a regulating role in heart rate. Since yoga training itself places a high priority on breathing regulation, insisting on breathing regulation exercises helps improve the body's autonomic nerve function, and at the same time strengthens the conversion of excitement and suppression between sympathetic and parasympathetic nerves. In addition, it can coordinate the adjustment mechanism to keep the sports center always in a 
benign state of excitement, so that the heart rate can be reduced and the recovery of the athlete's heart rate after training can be accelerated [13-14].

The subjective physical sensation degree is simple and effective for evaluating exercise intensity. This evaluation is actually a combination of physiological function and psychological perception [15]. From the 4th week to the 8th week, the observation group's subjective physical sensation degree score was lower than the control group $(P<0.05)$. It shows that the athletes in the observation group have better tolerance to the training intensity than the control group from the fourth week, indicating that the choice of yoga as a resuming training method can improve the athlete's physical strength. Because yoga practice emphasizes relaxation, slow movements can promote the recovery of body fatigue. In yoga practice, the self-perception ability can guide the body's relaxation, and continuous practice can let body learn to follow the body's wishes, which can speed up the relief of physical and mental fatigue [16]. In this study, many results of the observation group only began to show the difference from the control group at the fourth week. Since athletes have not contacted to yoga before, they are beginners. It is impossible to grasp the essence of yoga at the first contact, so it is impossible to fully gather energy during practice, which may affect the function of yoga. With the extension of the practice time, the athlete's mastery is improved and the degree of integration with yoga is higher, so the role of yoga can be gradually highlighted.

Hemoglobin levels reflect exercise endurance, and high hemoglobin levels suggest that athletes have more physical strength and are less prone to fatigue during training [17]. Athletes' blood urea nitrogen level will increase early in a training cycle, and then slowly return to the normal range. This change suggests that there is enough stimulation of the exercise load, and the body can adapt well [18]. If the athlete's blood urea nitrogen continues to rise during training, there is no tendency to decrease at all, meaning that his body's function has not been restored completely. In this case, the rest of the athlete needs to be increased. Creatine kinase can reflect the slight damage caused by skeletal muscle. The fluctuation of this index level is related to the amount and the intensity of training. Excessive exercise load stimulation will cause creatine kinase to increase, while at rest, creatine kinase will decrease [19]. The results of this study showed that after 8 weeks of resuming training, the observation group had higher hemoglobin than the control group, and blood urea nitrogen and creatine kinase was lower than the control group $(P<0.05)$. This means that the athletes in the observation group have stronger exercise endurance, and their bodies can recover more quickly and completely after training. The study found that girls in sports and dance majors who received yoga resuming training after training had better recovery quality than natural recovery, and athletes had stronger ankle flexor and extensor muscle groups after fatigue [20]. After training, football players choose yoga as resuming training. The results show that the players' relaxation experience and autonomous physiological response are better than the natural recovery group [21].

Taekwondo athletes with good flexibility have higher movement amplitude and movement quality when participating in training and formal competitions, and the attack strength of the movements is stronger, and the athletes with better flexibility are more likely to restore body function after exercise [22]. In this 
study, after 4 weeks and 8 weeks of resuming training, the sitting anteflexion test results of the observation group were higher than those of the control group $(P<0.05)$. It shows that the hip joint flexibility level of the observation group receiving yoga resuming training is increased more. The higher the flexibility of the hip joint of the athlete, the greater the action torque and the greater the completion of the action, and the more avoidance and attack can be completed in the same time. The function of yoga to improve flexibility is as follows: Yoga posture movements include stretching, bending, pushing, twisting, and fully stretching the muscles of the body through various asanas. Continuous practice can effectively increase the stretchability of athletes' ligaments and exercise muscles, so it can promote the flexibility of athletes [23-24]. Studies have suggested that the static stretch formed after exercise can speed up the recovery of skeletal muscle structure, strengthen the deformability of skeletal muscle, improve the flexibility of the body, thereby helping to reduce the risk of sports injuries, and also help restore the function after training [25].

In summary, the use of yoga for resuming training after high-intensity training can improve the physical function and physical fitness of Taekwondo athletes, enhance the flexibility of athletes, and promote professional skills. However, this study included fewer subjects, and only selected male taekwondo athletes from the Henan Taekwondo team. In addition to the shorter research time, the results obtained may be somewhat biased, lacking sufficient scientificity and representativeness. In the future, a longer period of time and a larger amount of studies should be carried out to further verify the value of yoga in the resuming training of athletes.

\section{Abbreviations}

\begin{tabular}{|lll|}
\hline English Abbreviation & Full Name in English & Full Name in Chinese \\
\hline BMI & Body Mass Index & Body Mass Index \\
\hline TKD & TeaKwonDo & TeaKwonDo \\
\hline HR & Heart Rate & Heart Rate \\
\hline Hb & Hemoglobin & Hemoglobin \\
\hline BUN & Blood Urea Nitrogen & Blood Urea Nitrogen \\
CK & Creatine Kinase & Creatine Kinase \\
\hline RPE & Rating of Perceived Exertion & Rating of Perceived Exertion \\
\hline
\end{tabular}

\section{Declarations}

\section{Ethics approval and consent to participate}

This article has clearly informed the research subjects of the background, purpose, content, process, possible risks, powers and privacy protection of the research subjects in the research. And has obtained 
the informed consent of all research subjects, and they signed the consent form voluntarily. In addition, the research has been approved by the hospital ethics committee before the start of the study.

\section{Consent for publication}

This article has not been submitted to other magazines in the past, and I agree to publish it in your magazine. The authors listed in this article agree to all the content and conclusions in this article, agree and promise the above content.

\section{Availability of data and materials}

The materials and data involved in this article are all obtained from previous research, and the materials and data obtained are approved for use by relevant units and research subjects. And promise not to include the research results of others.

\section{Competing interests}

The research results I submitted are sponsored by our unit, and the results belong to the unit.

\section{Funding}

All funds for this research are from the project of The Impact of Participating in Sports on People, Social, and Government Trust and Chinese martial arts and Traditional Culture and National Traditional Sports that provided funding, which project number are 2012 YBRW013 and 05YBRW041.

\section{Authors' Contributions}

I have done sufficient theoretical and practical work in data collection, research method combing, observation index analysis, statistical data, etc., and then provided indispensable value and contribution to this research.

\section{Acknowledgement}

For the completion of this paper, thanks to all research objects, foundations, contract units, enterprises, organizations and individuals who funded the research work. Thanks to colleagues who assisted in completing the research work, including but not limited to those who assisted in the completion of experiments or on-site surveys, and those who assisted in data compilation and statistical analysis. Thanks to the organizations and individuals who have facilitated the research work. Thanks to the 
owners of the materials, pictures, documents, and research ideas that have been given the right to reprint and quote. Thanks to the people who reviewed and helped modify the article. Thanks to the project of The Impact of Participating in Sports on People, Social, and Government Trust and Chinese martial arts and Traditional Culture and National Traditional Sports that provided funding, which project number are 2012 YBRW013 and 05YBRW041. Thanks to those who made suggestions during the research.

\section{References}

1. Yang JS, Ko JM, Roh HT. Effects of regular Taekwondo exercise on mood changes in children from multicultural families in South Korea: a pilot study[J]. J Phys Ther Sci. 2018;30(4):496-499.

2. Moscatelli F, Valenzano A, Petito A, et al. Relationship between blood lactate and cortical excitability between taekwondo athletes and non-athletes after hand-grip exercise[J]. Somatosens Mot Res. 2016;33(2):137-144.

3. Kaya O. Effect of a four-week exercise program on the secretion of IFN- $-\gamma$, TNF-a, IL-2 and IL-6 cytokines in elite Taekwondo athletes[J]. Biomed Rep. 2016;5(3):367-370.

4. Bridge CA, Sparks AS, McNaughton LR, et al. Repeated Exposure to Taekwondo Combat Modulates the Physiological and Hormonal Responses to Subsequent Bouts and Recovery Periods[J]. J Strength Cond Res. 2018;32(9):2529-2541.

5. Kim DY, Seo BD, Choi PA. Influence of taekwondo as security martial arts training on anaerobic threshold, cardiorespiratory fitness, and blood lactate recovery[J]. J Phys Ther Sci. 2014;26(4):471474.

6. Youkhana S, Dean $\mathrm{CM}$, Wolff $\mathrm{M}$, et al. Yoga-based exercise improves balance and mobility in people aged 60 and over: a systematic review and meta-analysis[J]. Age Ageing. 2016;45(1):21-29.

7. Vardar Yağlı N, Şener G, Arıkan H, et al. Do yoga and aerobic exercise training have impact on functional capacity, fatigue, peripheral muscle strength, and quality of life in breast cancer survivors? [J]. Integr Cancer Ther. 2015;14(2):125-132.

8. Morishita S, Tsubaki A, Nakamura M, et al. Rating of perceived exertion on resistance training in elderly subjects[J]. Expert Rev Cardiovasc Ther. 2019;17(2):135-142.

9. Plews DJ, Laursen PB, Stanley J, et al. Training adaptation and heart rate variability in elite endurance athletes: opening the door to effective monitoring[J]. Sports Med. 2013;43(9):773-781.

10. Leicht AS, Halliday A, Sinclair WH, et al. Heart rate variability responses to acute and repeated postexercise sauna in trained cyclists[J]. Appl Physiol Nutr Metab. 2018;43(7):704-710.

11. Plews $D J$, Laursen $P B$, Kilding $A E$,et al. Heart-rate variability and training-intensity distribution in elite rowers[J]. Int J Sports Physiol Perform. 2014;9(6):1026-1032.

12. Knoepfli-Lenzin C, Haenggli B, Boutellier U. Optimised heart rate formulae to monitor endurance training in sedentary individuals[J]. J Sports Sci. 2014;32(6):557-562.

13. Monroe DC, Yin J, McCully KK, et al. Yoga Aids Blood Pressure Recovery After Exposure of Forehead to Cold: A Pilot Study[J]. Altern Ther Health Med. 2018;24(5):12-17. 
14. Neyaz O, Sumila L, Nanda S, et al. Effectiveness of Hatha Yoga Versus Conventional Therapeutic Exercises for Chronic Nonspecific Low-Back Pain[J]. J Altern Complement Med. 2019;25(9):938-945.

15. Slimani M, Davis P, Franchini E, et al. Rating of Perceived Exertion for Quantification of Training and Combat Loads During Combat Sport-Specific Activities: A Short Review[J]. J Strength Cond Res. 2017;31(10):2889-2902.

16. Duma $\mathrm{S}$. Is exercise and yoga a panacea for recovery from sexual violence?: knowing the risks and benefits of yoga and exercise is important[J]. Evid Based Nurs. 2019;22(2):56.

17. Pedlar CR, Brugnara C, Bruinvels G, et al. Iron balance and iron supplementation for the female athlete: A practical approach[J]. Eur J Sport Sci. 2018;18(2):295-305.

18. Wankhede S, Mohan V, Thakurdesai P. Beneficial effects of fenugreek glycoside supplementation in male subjects during resistance training: A randomized controlled pilot study [J]. J Sport Health Sci. 2016;5(2):176-182.

19. Hunkin SL, Fahrner B, Gastin PB. Creatine kinase and its relationship with match performance in elite Australian Rules footbal[J]l. J Sci Med Sport. 2014;17(3):332-336.

20. Cramer H, Quinker D, Schumann D, et al. Adverse effects of yoga: a national cross-sectional survey[J]. BMC Complement Altern Med. 2019;19(1):190.

21. Kavak F, Ekinci M. The Effect of Yoga on Functional Recovery Level in Schizophrenic Patients[J]. Arch Psychiatr Nurs. 2016;30(6):761-767.

22. Cho SY, Roh HT. Taekwondo Enhances Cognitive Function as a Result of Increased Neurotrophic Growth Factors in Elderly Women[J]. Int J Environ Res Public Health. 2019;16(6):962.

23. Wang Y, Lu S, Wang R, et al. Integrative effect of yoga practice in patients with knee arthritis: $A$ PRISMA-compliant meta-analysis[J]. Medicine (Baltimore). 2018;97(31):e11742.

24. Loudon A, Barnett T, Williams A. Yoga, breast cancer-related lymphoedema and well-being: A descriptive report of women's participation in a clinical trial[J]. J Clin Nurs. 2017;26(23-24):46854695.

25. Gaihre A, Rajesh SK. Effect of Add-On Yoga on Cognitive Functions among Substance Abusers in a Residential Therapeutic Center: Randomized Comparative Study[J]. Ann Neurosci. 2018;25(1):38-45.

\section{Figures}




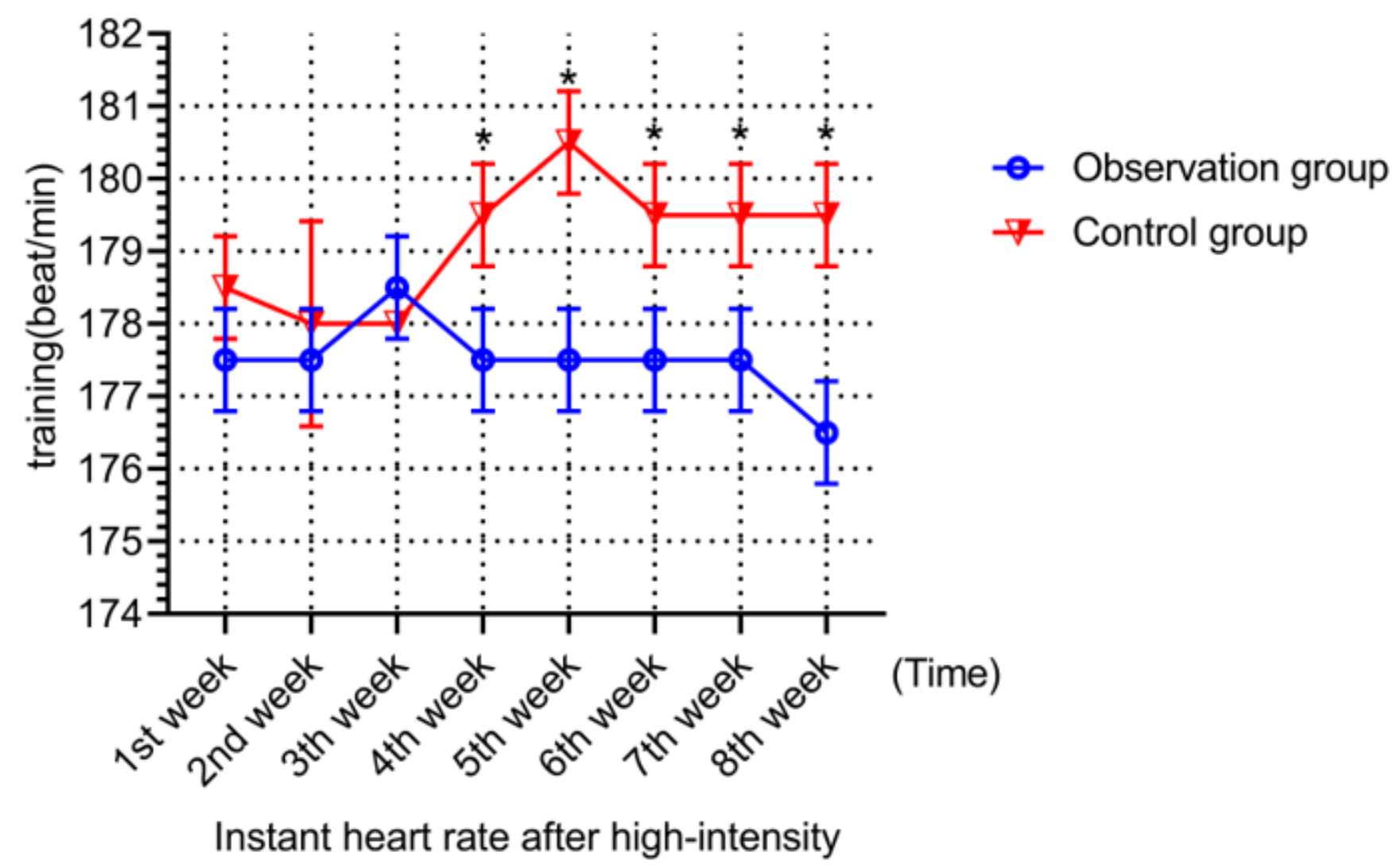

Figure 1

Comparison of instant heart rate between two groups after high-intensity training. 


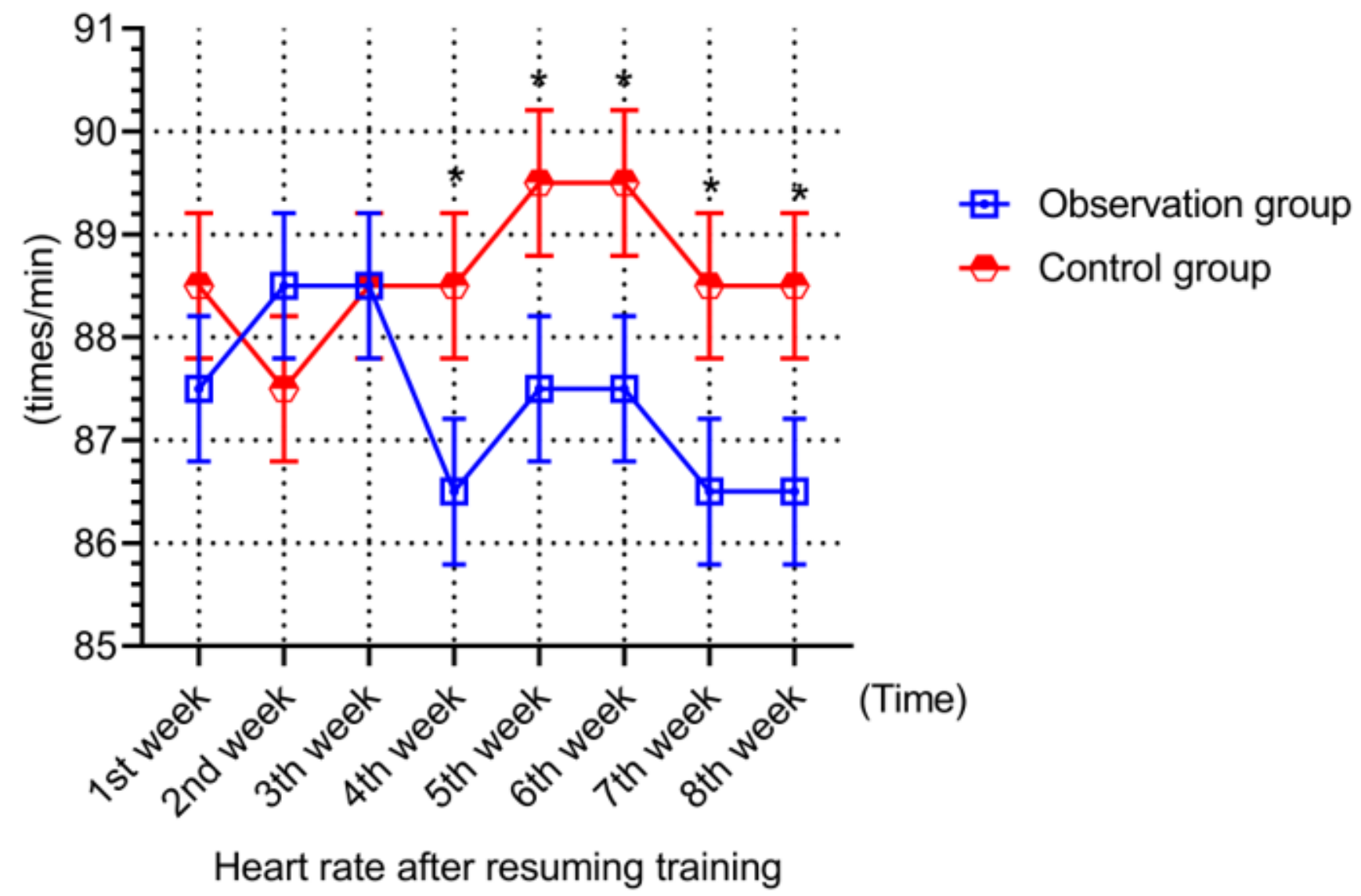

Figure 2

Comparison of heart rate after resuming training between the two groups 


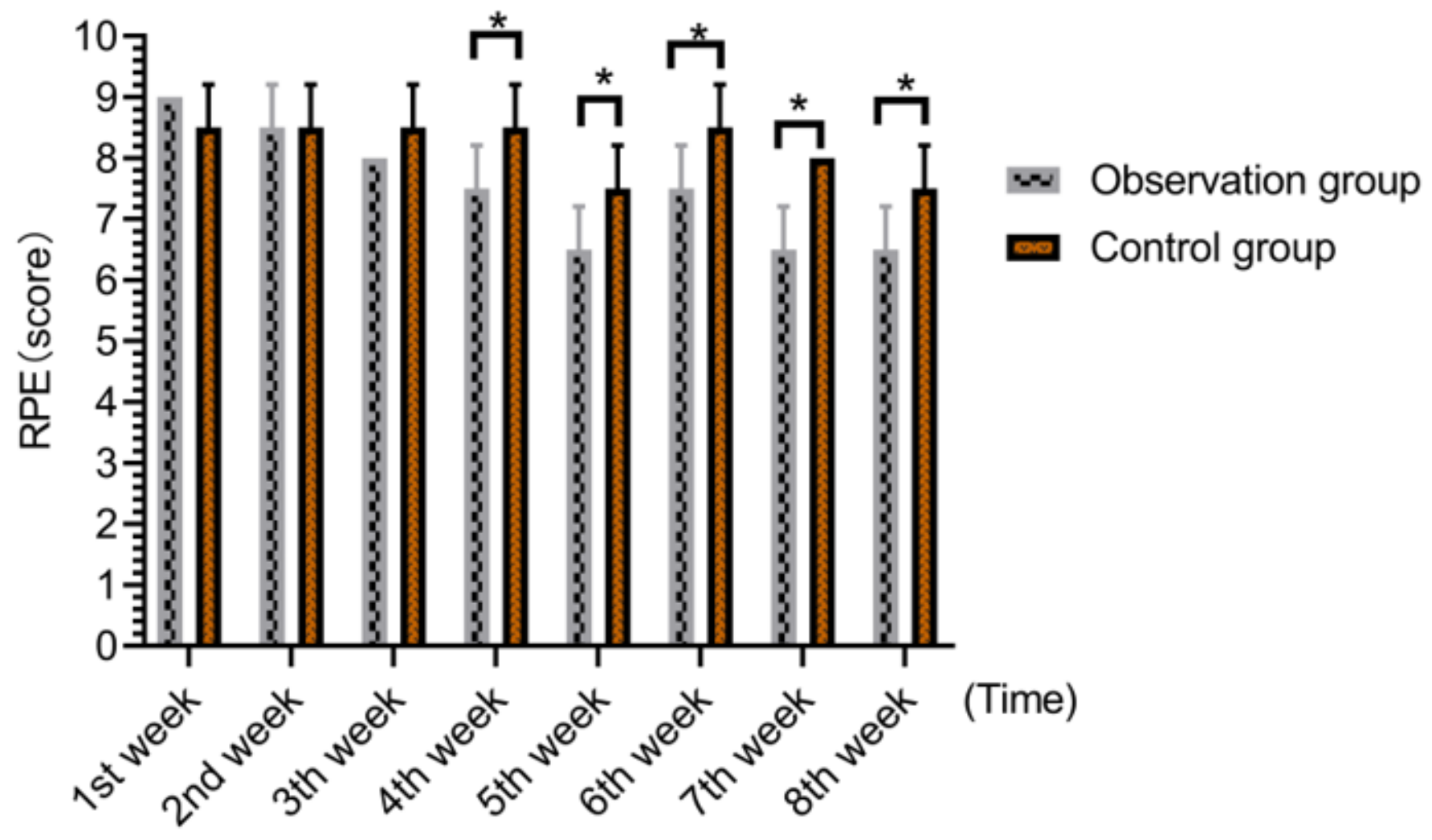

Figure 3

Comparison of subjective physical sensation between the two groups 

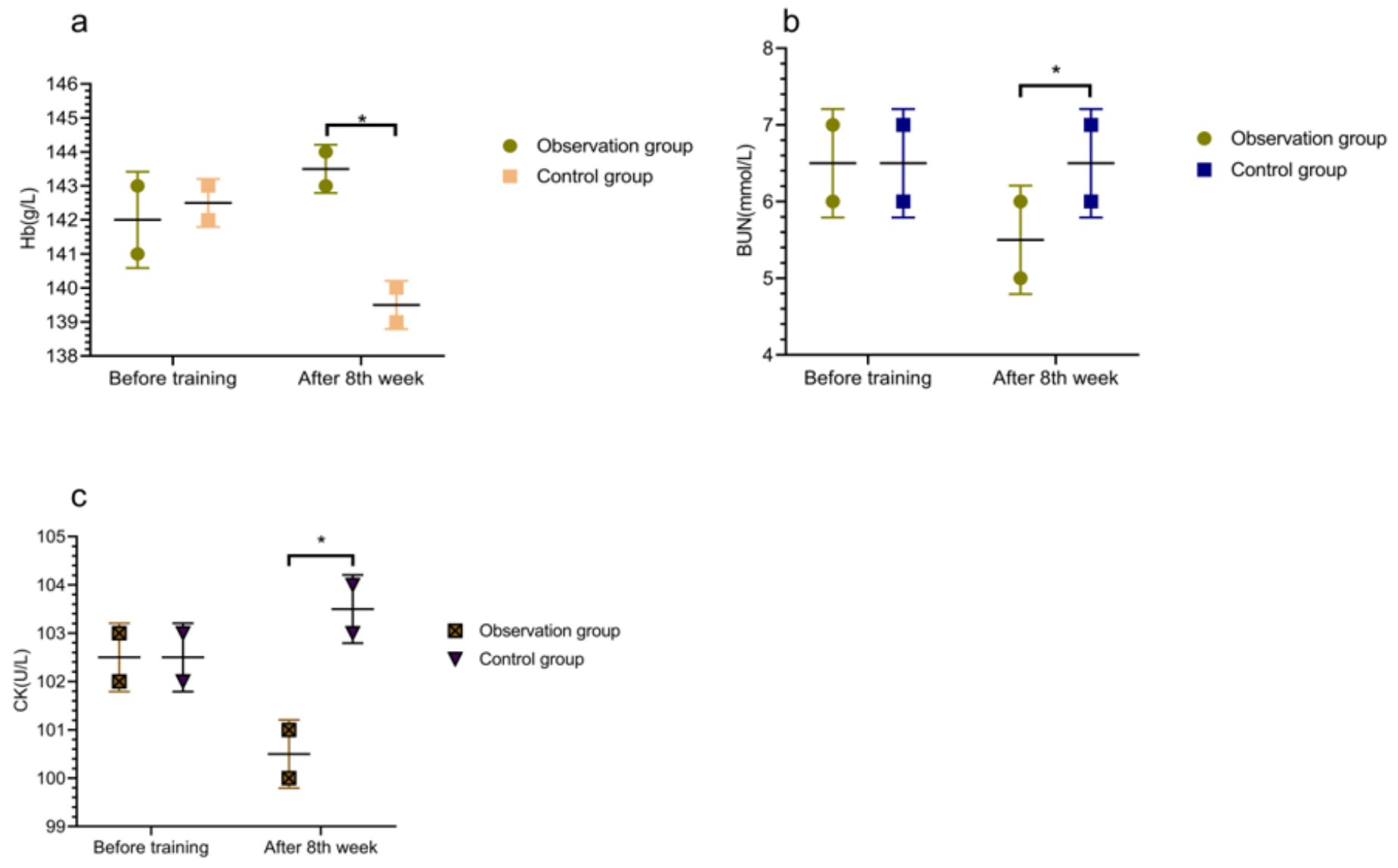

Figure 4

Comparison of biochemical index of two groups 